1. Marcel, A. J. Cognit. Psychol. 15, 197-237 (1983).

2. Dehaene, S. et al. Nat. Neurosci. 4, 752-758 (2001).

3. Desimone, R. Proc. Natl. Acad. Sci. USA 93, 13494-13499 (1996).

4. Rees, G. et al. Brain 123, 1624-1633 (2000).

5. Vuilleumier, P. et al. Proc. Natl. Acad. Sci. USA 98, 3495-3500 (2001).

6. Goebel, R., Muckli, L., Zanella, F. E., Singer, W. \& Stoerig, P. Vision Res. 41, 1459-1474 (2001).
7. Beck, D., Rees, G., Frith, C. D. \& Lavie, N. Nat. Neurosci. 4, 645-650 (2001).

8. Frith, C. D., Perry, R. \& Lumer, E. Trends Cogn. Sci. 3, 105-114 (1999).

9. Kleinschmidt, A., Buechel, C., Zeki, S. \& Frackowiak, R. S. J. Proc. R. Soc. Lond. B Biol. Sci. 265, 2427-2433 (1998).

10. Lumer, E. D., Friston, K. J. \& Rees, G. Science 280, 1930-1934 (1998)

11. Lumer, E. D. \& Rees, G. E. Proc. Natl. Acad. Sci.
USA 96, 1669-1673 (1999).

12. Portas, C. M., Strange, B. A., Friston, K. J., Dolan, R. J. \& Frith, C. D. Proc. R. Soc. Lond. B Biol. Sci. 267, 845-850 (2000).

13. Baars, B. A Cognitive Theory of Consciousness (Cambridge Univ. Press, Cambridge, 1988).

14. Crick, F. \& Koch, C. Nature 375, 121-123 (1995).

15. Treisman, A. M. \& Gelade, G. Cognit. Psychol. $12,97-136(1980)$.

\title{
Boundaries on motion integration
}

Visual perceptual processes occur with an accuracy and ease that belies the difficulty of the problems they solve, and the perception of object motion is no exception. On page 745 of this issue, Jean Lorenceau and David Alais shed new light on how we perceive object motion, showing that an object's shape exerts a powerful influence on motion integration, the process by which ambiguous local motion measurements are combined to determine the object's motion. A simple diamond, translating horizontally (left), illustrates the problem. Receptive fields of neurons in the visual cortex sample small regions of space, and those situated on a moving object's edge cannot determine the object's true motion. Although the diamond moves horizontally, an edge viewed in isolation (through the circle) appears to move diagonally, and is consistent with a wide range of object motions. Yet even when the unambiguous motions of the corners are covered with occluding surfaces (center), leaving only the edges, the diamond still appears to move rightward. The only way to derive rightward motion from this stimulus is to combine motion information across edges. Each edge alone is ambiguous, but together, they yield the correct motion direction. Much research now supports the idea that local motion measurements are integrated across space in this manner.

The integration process is tricky, however. Most scenes contain more than one object, and if edge motions are combined across objects, the resulting motion estimates will be wrong. The visual system must somehow restrict motion integration to measurements made on the same object. Lorenceau and Alais investigate this process by permuting the contours in their stimuli. In the example on the right, the top and bottom contours of the diamond have simply been swapped. The motion information is thus unchanged, but the new contours form a different global shape. This simple manipulation has a drastic effect on motion perception. Whereas the original diamond configuration is perceived to move as a single solid object, the contours in the new configuration are almost always seen to move independently. Thus the visual system opts not to integrate the edge motions even though they are perfectly consistent with a single global motion. What accounts for the difference? One critical variable seems to be contour closure. The authors found that contour configurations that did not form a closed shape were almost never seen to cohere. The unclosed shapes remained incoherent even after subjects were shown the full, unoccluded shape (in this case, a bow-tie). The visual system thus seems to have a built-in bias favoring integration for closed shapes. Given that objects in the world tend to have closed bounding contours, these influences of shape on motion interpretation may serve to avoid integrating across different objects.

\section{Josh McDermott}
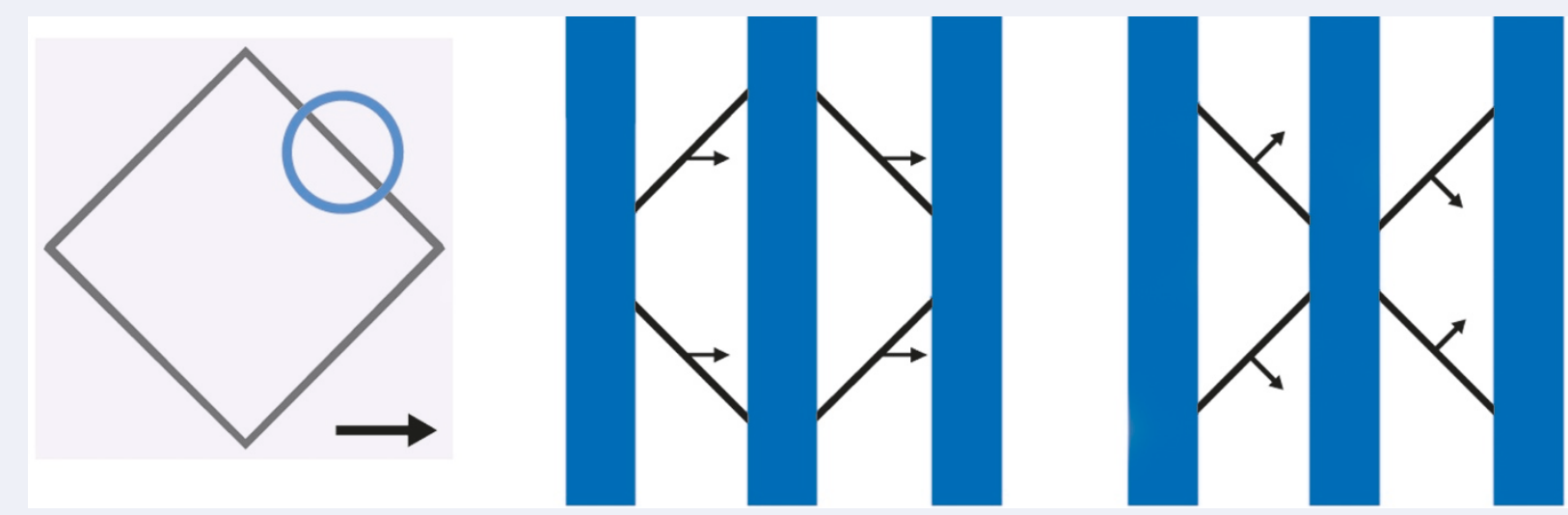